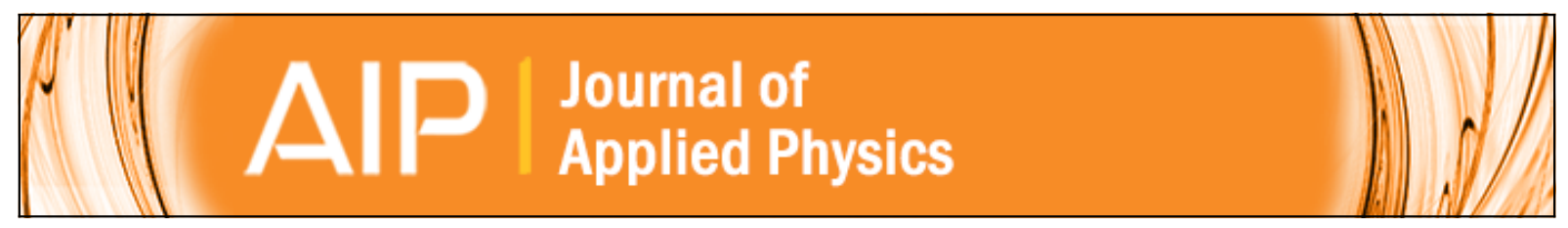

\title{
Self-biased magnetoelectric response in three-phase laminates
}

Su-Chul Yang, Chee-Sung Park, Kyung-Hoon Cho, and Shashank Priya

Citation: Journal of Applied Physics 108, 093706 (2010); doi: 10.1063/1.3493154

View online: http://dx.doi.org/10.1063/1.3493154

View Table of Contents: http://scitation.aip.org/content/aip/journal/jap/108/9?ver=pdfcov

Published by the AIP Publishing

\section{Articles you may be interested in}

Self-biased large magnetoelectric coupling in co-sintered $\mathrm{Bi0.5Na0.5TiO3}$ based piezoelectric and $\mathrm{CoFe} 2 \mathrm{O} 4$ based magnetostrictive bilayered composite

J. Appl. Phys. 116, 244101 (2014); 10.1063/1.4904758

Giant self-biased converse magnetoelectric effect in multiferroic heterostructure with single-phase magnetostrictive materials

Appl. Phys. Lett. 105, 172408 (2014); 10.1063/1.4900929

Giant self-biased magnetoelectric coupling in co-fired textured layered composites

Appl. Phys. Lett. 102, 052907 (2013); 10.1063/1.4791685

Self-biased converse magnetoelectric effect

Appl. Phys. Lett. 99, 202904 (2011); 10.1063/1.3662420

Magnetoelectric effect in lead-free BNKLBT ceramic/terfenol-D continue fiber composite laminates J. Appl. Phys. 107, 093907 (2010); 10.1063/1.3385413

MIT LINCOLN LABORATORY CAREERS

Discover the satisfaction of innovation and service to the nation
- Space Control

- Air \& Missile Defense

- Communications Systems \& Cyber Security

- Intelligence, Surveillance and

Reconnaissance Systems
- Advanced Electronics

- Tactical Systems

- Homeland

Protection

- Air Traffic Control

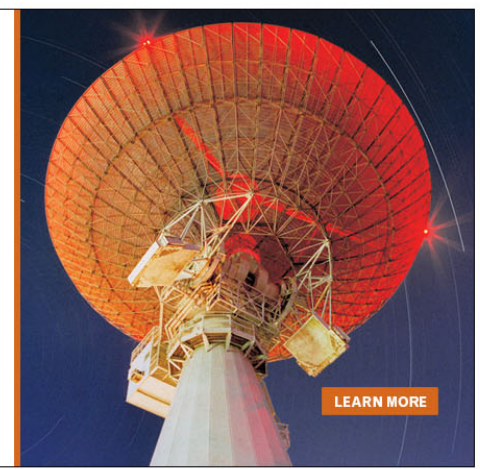




\title{
Self-biased magnetoelectric response in three-phase laminates
}

\author{
Su-Chul Yang, Chee-Sung Park, Kyung-Hoon Cho, and Shashank Priya ${ }^{\text {a) }}$ \\ Department of Materials Science and Engineering, Center for Energy Harvesting Materials and Systems \\ (CEHMS), Virginia Polytechnic Institute and State University, Blacksburg, Virginia 24061, USA
}

(Received 16 June 2010; accepted 25 August 2010; published online 3 November 2010)

\begin{abstract}
This study reports the experimental observation and analysis of self-biased magnetoelectric (ME) effect in three-phase laminates. The 2-2 L-T mode laminates were fabricated by attaching nickel (Ni) plates and $\mathrm{ME}$ particulate composite plates having 3-0 connectivity with $0.948 \mathrm{Na}_{0.5} \mathrm{~K}_{0.5} \mathrm{NbO}_{3}-0.052 \mathrm{LiSbO}_{3}(\mathrm{NKNLS})$ matrix and $\mathrm{Ni}_{0.8} \mathrm{Zn}_{0.2} \mathrm{Fe}_{2} \mathrm{O}_{4}$ (NZF) dispersant. The presence of two types of ferromagnetic materials, Ni and NZF, results in built-in magnetic bias due to difference in their magnetic susceptibilities and coercivity. This built-in bias $\left(\mathrm{H}_{\text {bias }}\right)$ provides finite ME effect at zero applied magnetic dc field. The ME response of bending mode trilayer laminate NKNLS-NZF/Ni/NKNLS-NZF in off-resonance and on-resonance conditions was shown to be mathematical combination of the trilayers with configuration NKNLS-NZF/Ni/NKNLS-NZF and NKNLS/Ni/NKNLS representing contributions from magnetic interaction and bending strain. (C) 2010 American Institute of Physics. [doi:10.1063/1.3493154]
\end{abstract}

\section{INTRODUCTION}

Multiferroic magnetoelectric (ME) materials have coexistence of ferroelectric and ferromagnetic order which makes them attractive for magnetic field sensors, tunable transformers, and memory devices. ${ }^{1-5}$ It is well known that ME effect in 3-0 particulate composites is small but 2-2 laminate composites have been shown to provide giant enhancement in the magnitude of ME coefficient by optimizing the properties of individual piezoelectric and magnetostrictive phases. ${ }^{6,7} \mathrm{ME}$ coefficient is dependent upon the magnetoelastic and elastoelectric interactions and is favored in composites with high elastic compliance. Most of the reported ME composites in literature require magnetic dc bias to invoke piezomagnetic response and only recently, Mandal et al. demonstrated selfbiased laminate composites where ME response at zero field was correlated with presence of flexural deformation in a compositionally graded structure. ${ }^{8-10}$ However, this method of generating self-bias is dependent upon the composition grading and requires special synthesis process. Our research has been focused on finding a methodology where we can achieve self-biasing by just changing the electrical connections with regular laminate composites. In this study, we report the success in demonstrating the self-biased ME effect in three-phase laminates having "sandwich" structure, with $\mathrm{Ni}$ embedded between the $0.8\left[0.948 \mathrm{Na}_{0.5}\right.$ $\left.\mathrm{K}_{0.5} \mathrm{NbO}_{3}-0.052 \mathrm{LiSbO}_{3}\right]-0.2\left[\mathrm{Ni}_{0.8} \mathrm{Zn}_{0.2} \mathrm{Fe}_{2} \mathrm{O}_{4}\right]$ (NKNLSNZF) ME particulate composite layers. This three-phase composite consists of lead-free materials which is critical for the applications. ${ }^{11-14}$

\section{EXPERIMENTAL}

$0.948 \mathrm{Na}_{0.5} \mathrm{~K}_{0.5} \mathrm{NbO}_{3}-0.052 \mathrm{LiSbO}_{3} \quad$ (NKNLS) and $\mathrm{Ni}_{0.8} \mathrm{Zn}_{0.2} \mathrm{Fe}_{2} \mathrm{O}_{4}$ (NZF) ceramics were synthesized by mixed oxide sintering method. ${ }^{13,15}$ NKNLS-NZF/Ni bilayer and

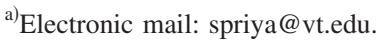

NKNLS-NZF/Ni/NKNLS-NZF trilayer laminates were fabricated by bonding $\mathrm{Ni}$ plates with dimension of 15 $\times 15 \mathrm{~mm}^{2}$ with NKLNS-NZF disks of diameter $10 \mathrm{~mm}$, using epoxy with curing temperature of $80{ }^{\circ} \mathrm{C}$. Each layer had a thickness of $0.5 \mathrm{~mm}$ and the $\mathrm{ME}$ composite operated in L-T mode. Magnetic properties of the laminate were measured by using vibrating sample magnetometer (VSM 7304, Lake Shore Cryotronics). The impedance spectrum of the laminates was measured by an LCR meter (HP4194A, USA). ME voltage constants of the laminates were measured by applying dc bias magnetic field with $\mathrm{H}_{\mathrm{ac}}=1$ Oe at $1 \mathrm{kHz}{ }^{16}$ The voltage induced on the laminates was monitored by using a lock-in amplifier.

\section{RESULTS AND DISCUSSION}

Figure 1 shows the M-H curves for NZF particles and Ni plates. NZF particles had saturation magnetization $\left(\mathrm{M}_{\mathrm{s}}\right)$ of $64.6 \mathrm{emu} / \mathrm{g}$ under an applied magnetic field of $3000 \mathrm{Oe}$. The magnetic susceptibility $(\chi)$ was calculated to be 3.3 $\times 10^{-2} \mathrm{emu} / \mathrm{g}$ Oe in the magnetic field range of $(-1000)-$ 1000 Oe, as shown in Fig. 1(a). Nickel plates were found to posses the saturation magnetization $\left(\mathrm{M}_{\mathrm{s}}\right)$ of $50.2 \mathrm{emu} / \mathrm{g}$ at an applied magnetic field of 4000 Oe. The magnetic susceptibility $(\chi)$ was calculated to be $1.6 \times 10^{-2} \mathrm{emu} / \mathrm{g}$ Oe in the magnetic field range of (-1000)-1000 Oe, as shown in Fig. 1(a). Using Fig. 1(b), the coercive field $\left(\mathrm{H}_{\mathrm{c}}\right)$ of NZF particles was found to be $5 \mathrm{Oe}$ and the $\mathrm{H}_{\mathrm{c}}$ for $\mathrm{Ni}$ was found to be 14 Oe. The remnant magnetizations $\left(\mathrm{M}_{\mathrm{r}}\right)$ for Ni and NZF particles was of the same amplitude, $0.29 \mathrm{emu} / \mathrm{g}$. These results indicate that the composite fabricated by using Ni and NZF phase will have built-in bias field $\left(\mathrm{H}_{\text {bias }}\right)$ which is related to difference in the magnitude of susceptibility and coercivity.

Figure 2 shows the ME voltage coefficient $\left(\alpha_{\mathrm{E}}\right)$ for bilayer and trilayer laminates. All the measurements in this figure were conducted at $1 \mathrm{kHz}$. It can be seen in Fig. 2(a) that NKNLS-NZF/Ni bilayer had maximum $\alpha_{\mathrm{E}}$ of $166 \mathrm{mV} / \mathrm{cm}$ Oe at an applied magnetic dc bias of $120 \mathrm{Oe}$ 

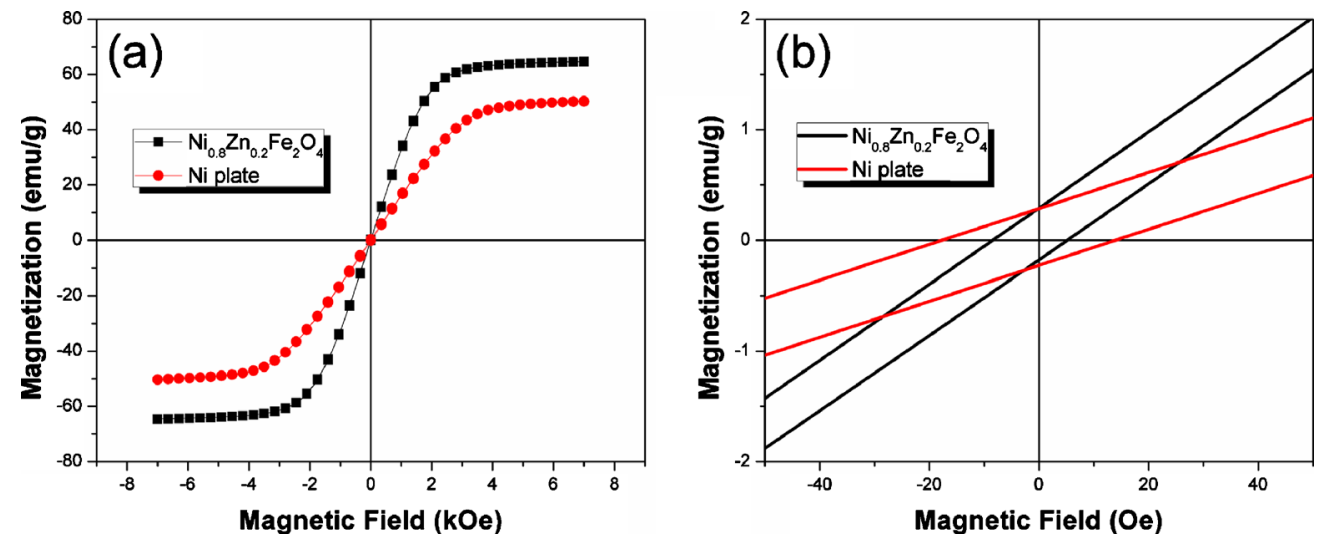

FIG. 1. (Color online) M-H curves for NZF particles and Ni plates, (a) saturated magnetic hysteresis loops under high field and (b) exploded view in the low field region.

and remanant ME coefficient $\alpha_{\mathrm{ER}}$ of $30 \mathrm{mV} / \mathrm{cm}$ Oe at zero magnetic dc bias. It should be noted here that in these structures, Ni plays three roles, as: (i) it acts as electrode, (ii) it induces bending modes in the composite at low frequencies by lowering the overall stiffness, and (iii) it magnetically couples with the NZF phase. In bilayer, there is structural asymmetry and mismatch of coercive force induced by differences in $\chi$ and $\mathrm{H}_{\mathrm{c}}$ of two ferromagnetic materials given by $\mathrm{H}_{\text {bias }}$. This asymmetry is further enhanced at higher magnetic dc bias owing to mismatch in the magnetostrictive strain of NZF and Ni. Thus, the ME response is summation of three effects, one related to the $\mathrm{H}_{\text {bias }}$, second related to the resultant of applied magnetic dc bias field $\left(\mathrm{H}_{\text {appl }}\right)$ and $\mathrm{H}_{\text {bias }}$ given as $\sum \overrightarrow{\mathrm{H}}_{\text {appl }}+\overrightarrow{\mathrm{H}}_{\text {bias }}$ which is frequency dependent term, and third related to structural asymmetry which induces bending modes. The bending strain is also frequency dependent term. The data in Figs. 2(b) and 2(c) further strengthens this argument. Figure 2(b) shows that NKNLS/Ni bilayer exhibited maximum $\alpha_{\mathrm{E}}$ of $120 \mathrm{mV} / \mathrm{cm}$ Oe at an applied magnetic dc bias of 150 Oe without any $\alpha_{\mathrm{ER}}$. The low $\alpha_{\mathrm{E}}$ and zero $\alpha_{\mathrm{ER}}$ could be related to absence of magnetic interaction between $\mathrm{Ni}$ and NZF. A comparison of Figs. 2(a) and 2(b) indicates that hysteresis is related to the magnetic interaction between $\mathrm{Ni}$ and NZF. The NZF phase was absent in Fig. 2(b) and the $\mathrm{ME}$ behavior was similar to that obtained for conventional laminates with linear response. The magnitude of $\alpha_{\mathrm{E}}$ for NKNLS-NZF/Ni/NKNLS-NZF trilayer exhibited maximum $\alpha_{\mathrm{E}}$ of $385 \mathrm{mV} / \mathrm{cm}$ Oe at an applied magnetic dc bias of 96 Oe without any $\alpha_{\mathrm{ER}}$ as shown in Fig. 2(c). This configuration in Fig. 2(c) will be referred to as "radial-mode trilayer (RMT)." High $\alpha_{\mathrm{E}}$ of trilayer and zero $\alpha_{\mathrm{ER}}$ was related to structural symmetry which reduces in-plane strain and maximizes the out-of-plane strain. ${ }^{8}$ The peak position in this curve corresponds with the maximum in gradient of the magnetostriction versus magnetic dc bias curve. Please note the electrical connection in Fig. 2(c) which will generate charge
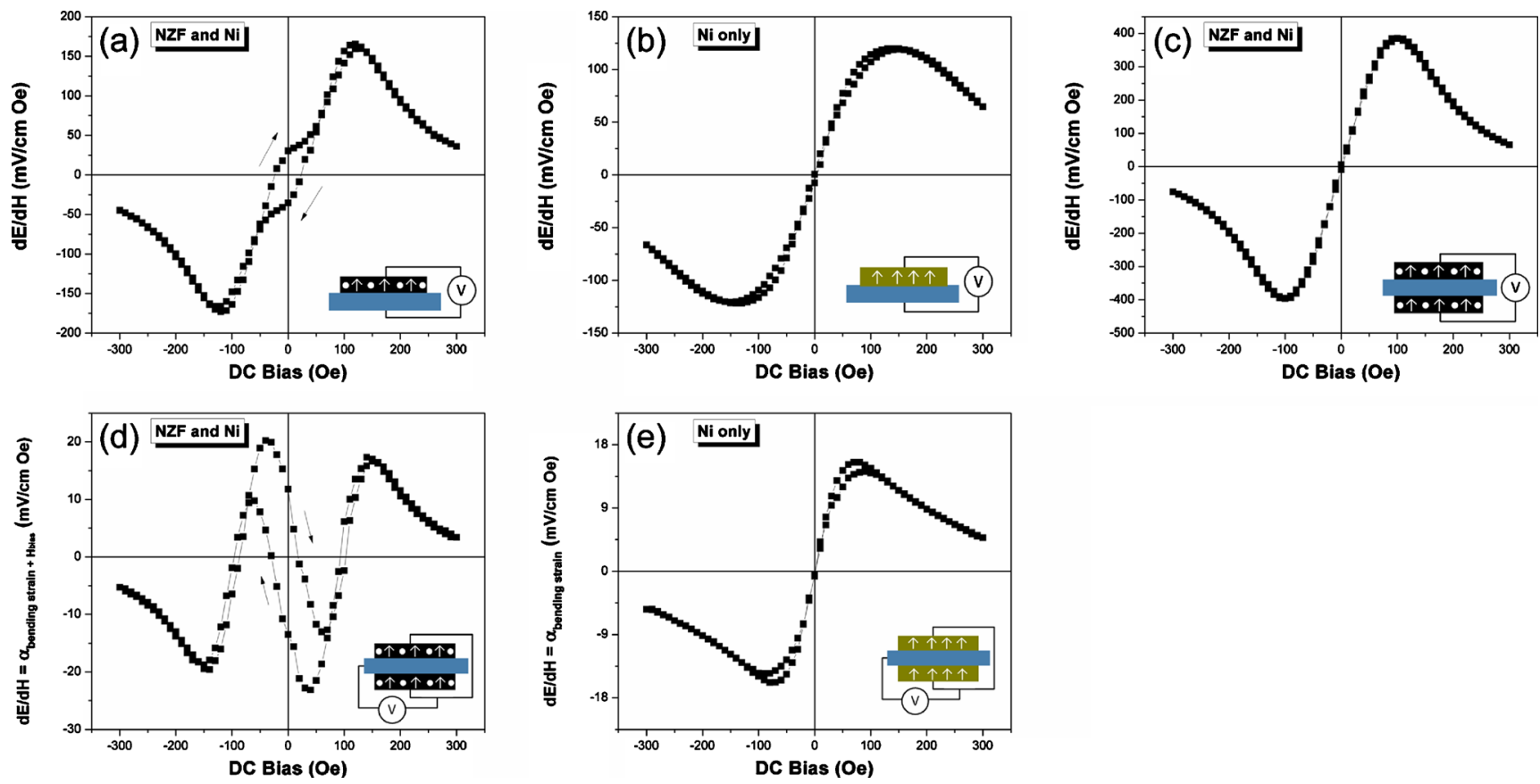

FIG. 2. (Color online) ME voltage coefficients $\left(\alpha_{\mathrm{E}}\right)$ for various laminates, (a) NKNLS-NZF/Ni bilayer, (b) NKNLS/Ni bilayer, (c) NKNLS-NZF/Ni/NKNLSNZF RMT, (d) NKNLS-NZF/Ni/NKNLS-NZF BMT, and (e) NKNLS/Ni/NKNLS trilayer. 

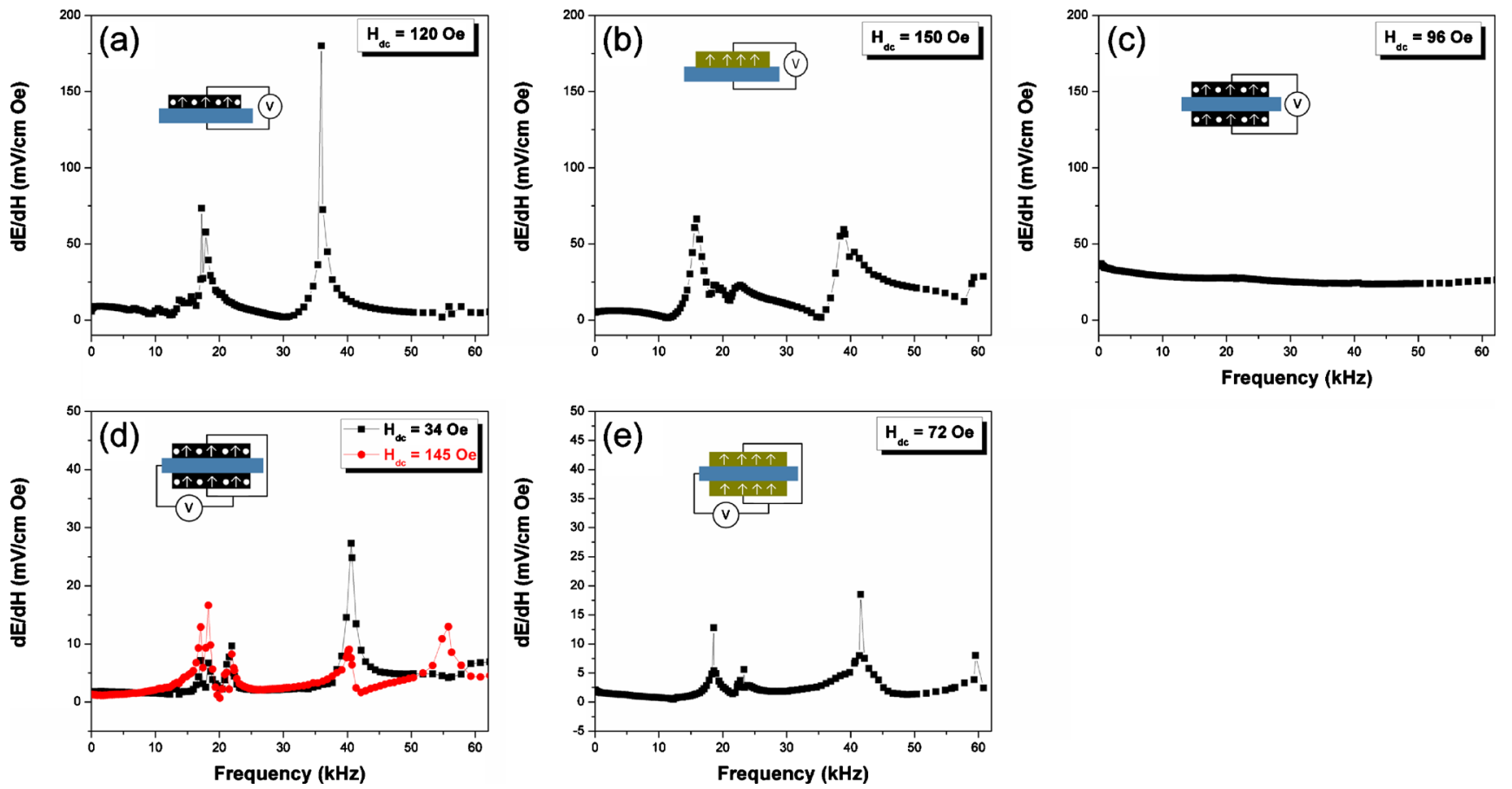

FIG. 3. (Color online) ME voltage coefficients $\left(\alpha_{\mathrm{E}}\right)$ for bilayer and trilayer laminates as a function of frequency from $0.1 \mathrm{ko} 60 \mathrm{kHz}$, (a) NKNLS-NZF/Ni bilayer, (b) NKNLS/Ni bilayer, (c) NKNLS-NZF/Ni/NKNLS-NZF RMT, (d) NKNLS-NZF/Ni/NKNLS-NZF bending-mode laminate, and (e) NKNLS/Ni/ NKNLS trilayer.

on the piezoelectric layer due to symmetric deformation in top and bottom layer. In contrast, Fig. 2(d) shows the ME response of NKNLS-NZF/Ni/NKNLS-NZF trilayer composite with modified electric connections to excite the bending mode, and will be referred as "bending mode trilayer (BMT)." In this case, there was clear hysteresis in the forward and backward sweep of ME response with zero-cross over points. The region defined by $-50<\mathrm{H}_{\mathrm{dc}}<50$ Oe represents the switchable states by changing the applied dc bias. In the forward sweep, the minimum in $\alpha_{\mathrm{E}}$ was found to occur at $\sim 16.6 \mathrm{mV} / \mathrm{cm} \mathrm{Oe}$ and maxima was found at $\sim 20 \mathrm{mV} / \mathrm{cm}$ Oe. In the backward sweep, the maxima was found at $23.1 \mathrm{mV} / \mathrm{cm}$ Oe with minimum occurring at $\sim 10 \mathrm{mV} / \mathrm{cm}$ Oe. The magnitude of $\alpha_{\mathrm{ER}}$ was $11.78 \mathrm{mV} / \mathrm{cm}$ Oe at zero de bias. BMT has clear $\alpha_{\mathrm{ER}}$ as well as two zero-cross over points at magnetic dc bias of 17.7 and 92.8 Oe. The behavior was not exactly symmetric in the positive and negative quadrants of applied magnetic field with slight shift toward negative Y-axis. We think that this shift is related to the magnitude of $\Sigma \overrightarrow{\mathrm{H}}_{\text {appl }}+\overrightarrow{\mathrm{H}}_{\text {bias }}$ which may have different magnitude during the forward and backward sweep depending upon the alignment of $\vec{H}_{\text {appl }}$ with $\overrightarrow{\mathrm{H}}_{\text {bias }}$.

In order to understand the ME response of Fig. 2(d), we present data on BMT without any NZF phase. Figure 2(e) shows the ME response of NKNLS/Ni/NKNLS trilayer without any NZF. A maximum $\alpha_{\mathrm{E}}$ of $15.5 \mathrm{mV} / \mathrm{cm}$ Oe was obtained at magnetic de bias field of 72 Oe without any $\alpha_{\mathrm{ER}}$. Further, the magnitude of $\alpha_{\mathrm{E}}$ was quite small as compared to ME behavior shown for RMT. This result clearly shows that interaction between NZF and Ni is important to achieve high $\alpha_{\mathrm{E}}$ and finite $\alpha_{\mathrm{ER}}$ with zero-cross over points. This also confirms our hypothesis that BMT ME response is governed by $\sum \overrightarrow{\mathrm{H}}_{\text {appl }}+\overrightarrow{\mathrm{H}}_{\text {bias }}$. Higher magnitude of $\alpha_{\mathrm{ER}}$ in Fig. 2(a) has some contribution from the lower stiffness of the bilayer structure. In order to illustrate the role of radial and bending vibration modes, we show the impedance and phase spectra in Ref. 17 (Fig. S1) for bilayer and trilayer laminates under a small applied ac electric field. It was found that NKNLS-NZF/Ni bilayer exhibited resonance peaks at 17.2, 35.9, and 55.8 $\mathrm{kHz}$ which were related to bending modes. NKNLS/Ni bilayer exhibited resonance peaks at $18.9,38.9$, and $60.0 \mathrm{kHz}$ which were related to bending modes due to structural asymmetry. This structure represents the conventional unimorph. However, NKNLS-NZF/Ni/NKNLS-NZF RMT did not exhibit any peaks in the same range. The impedance measurements indicate that RMT composite had dominant radial mode at low frequencies. BMT exhibited low frequency resonance peaks at $18.4,22.2,40.9,55.8$, and $58.3 \mathrm{kHz}$. BMT without any NZF, given by NKNLS/Ni/NKNLS also exhibited resonance peaks at 18.8, 22.5, 41.3, and $58.8 \mathrm{kHz}$. Thus, both the BMT composites shown in Figs. 2(d) and 2(e), exhibited bending resonance peaks but only the structure shown in Fig. 2(d) had the self-bias magnetic response with zero-cross over points. This result confirms that the selfbiased ME effect has prime contribution from the magnetic interaction between the NZF and $\mathrm{Ni}$, or $\mathrm{H}_{\text {bias }}$.

Figure 3 shows the variation in $\alpha_{\mathrm{E}}$ for bilayer and trilayer laminates as a function of frequency from 0.1 to 60 $\mathrm{kHz}$. ME response of NKNLS-NZF/Ni bilayer is shown in Fig. 3(a), exhibiting peaks at bending mode frequencies of $17.2,17.8$, and $35.9 \mathrm{kHz}$ under applied magnetic dc bias of $\mathrm{H}_{\mathrm{appl}}=120$ Oe with an superimposed ac magnetic field of 1 Oe. Figure 3(b) shows that NKNLS/Ni bilayer without any NZF phase exhibited peaks at bending mode frequencies of 16.0 and $38.9 \mathrm{kHz}$ under applied magnetic de bias of $\mathrm{H}_{\mathrm{appl}}$ 

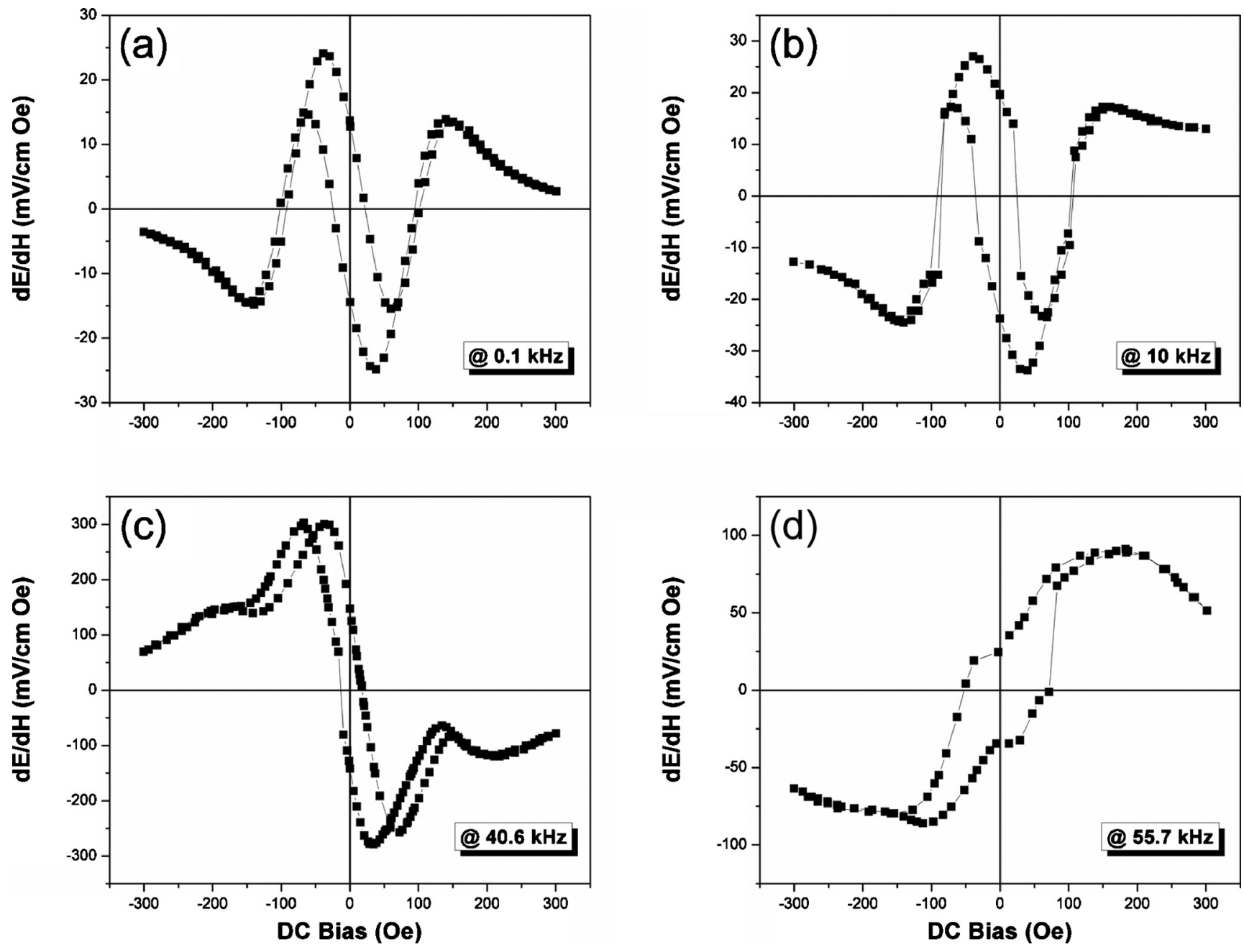

FIG. 4. ME voltage coefficients $\left(\alpha_{\mathrm{E}}\right)$ for bending mode NKNLS-NZF/Ni/NKNLS-NZF trilayer as a function of magnetic dc bias field at frequencies of (a) 0.1 $\mathrm{kHz}$, (b) $10 \mathrm{kHz}$, (c) $40.6 \mathrm{kHz}$, and (d) $55.7 \mathrm{kHz}$.

$=150$ Oe with an superimposed ac magnetic field of 1 Oe. In comparison to Fig. 3(a), the ME peak near $35 \mathrm{kHz}$ was reduced significantly in the absence of NZF. Thus, the ME peak at $35.9 \mathrm{kHz}$ in Fig. 3(a) is related to both magnetic interaction between $\mathrm{Ni}$ and NZF, and bending related to structural asymmetry. ME response of the RMT composite remained almost constant over the whole frequency range at magnetic dc bias of $\mathrm{H}_{\text {appl }}=96$ Oe as shown in Fig. 3(c). The magnitude of applied dc bias was determined from the ME peak obtained in Fig. 2 for the corresponding laminate structures. ME response of BMT exhibited peaks at frequencies of 17.0, 18.2, 21.9, and $40.6 \mathrm{kHz}$ under applied magnetic dc bias of $\mathrm{H}_{\text {appl }}=34 \mathrm{Oe}$, and 17.0, 18.2, 21.9, 40.2, and 55.7 $\mathrm{kHz}$ under magnetic dc bias of $\mathrm{H}_{\mathrm{appl}}=145$ Oe as shown in Fig. 3(d). Near $40.6 \mathrm{kHz}$, the ME response at magnetic dc bias of $\mathrm{H}_{\text {appl }}=34$ Oe was higher than that at magnetic dc bias of $\mathrm{H}_{\text {appl }}=145$ Oe. Further near $55.8 \mathrm{kHz}$, there is a peak in $\mathrm{ME}$ response at magnetic dc bias of $\mathrm{H}_{\mathrm{appl}}=145$ Oe which was not observed at magnetic dc bias of $\mathrm{H}_{\text {appl }}=34$ Oe. This result shows that in BMT composite the magnitude of $\mathrm{H}_{\text {bias }}$ changes with frequency and confirms the hypothesis that the magnitude of summation $\Sigma \overrightarrow{\mathrm{H}}_{\text {appl }}+\overrightarrow{\mathrm{H}}_{\text {bias }}$ determines the magnitude of $\alpha_{\mathrm{E}}$. The ME response of BMT without any NZF phase exhibited peaks at frequencies of 18.5, 23.3, 41.5, and
$59.5 \mathrm{kHz}$ under magnetic dc bias of $\mathrm{H}_{\text {appl }}=72$ Oe, as shown in Fig. 3(e). These peak ME frequencies are related to bending only, quite similar to that obtained for BMT with NZF phase at $\mathrm{H}_{\mathrm{dc}}=145$ Oe in Fig. 3(d). Therefore, we can determine that magnetic interaction dominates the ME response in BMT with NZF phase at $\mathrm{H}_{\mathrm{dc}}=35 \mathrm{Oe}$ and bending strain dominates the ME response in BMT with NZF phase at $\mathrm{H}_{\mathrm{dc}}=134$ Oe.

Figure 4 shows the ME voltage coefficient for BMT as a function of magnetic dc bias at frequencies of 0.1, 10, 40.6, and $55.7 \mathrm{kHz}$ under applied ac magnetic field of 1 Oe. At the nonresonance frequencies of 0.1 and $10 \mathrm{kHz}$, BMT has small remanant $\alpha_{\mathrm{ER}}$ and two zero-cross over points of $\alpha_{\mathrm{E}}$, as shown in Figs. 4(a) and 4(b). Further, at frequency of 10 $\mathrm{kHz}, \mathrm{BMT}$ has wider hysteresis area than that of BMT at the frequency of $0.1 \mathrm{kHz}$. At the resonance frequency of 40.6 $\mathrm{kHz}$ in Fig. 4(c), BMT has high remanant $\alpha_{\mathrm{ER}}$ of $147.3 \mathrm{mV} / \mathrm{cm}$ Oe and one zero-cross over point of $\alpha_{\mathrm{E}}$. This data illustrates that increased ME coefficient at low dc bias magnetic field of 34.5 Oe leads to one zero-cross over point of $\alpha_{\mathrm{E}}$ by reducing relative change in $\mathrm{ME}$ coefficient at high dc bias magnetic field of 134.2 Oe. At the resonance frequency of $59.5 \mathrm{kHz}$, BMT has remanant $\alpha_{\mathrm{ER}}$ of $24.4 \mathrm{mV} / \mathrm{cm} \mathrm{Oe}$ and one zero-cross over point of $\alpha_{\mathrm{E}}$, as 

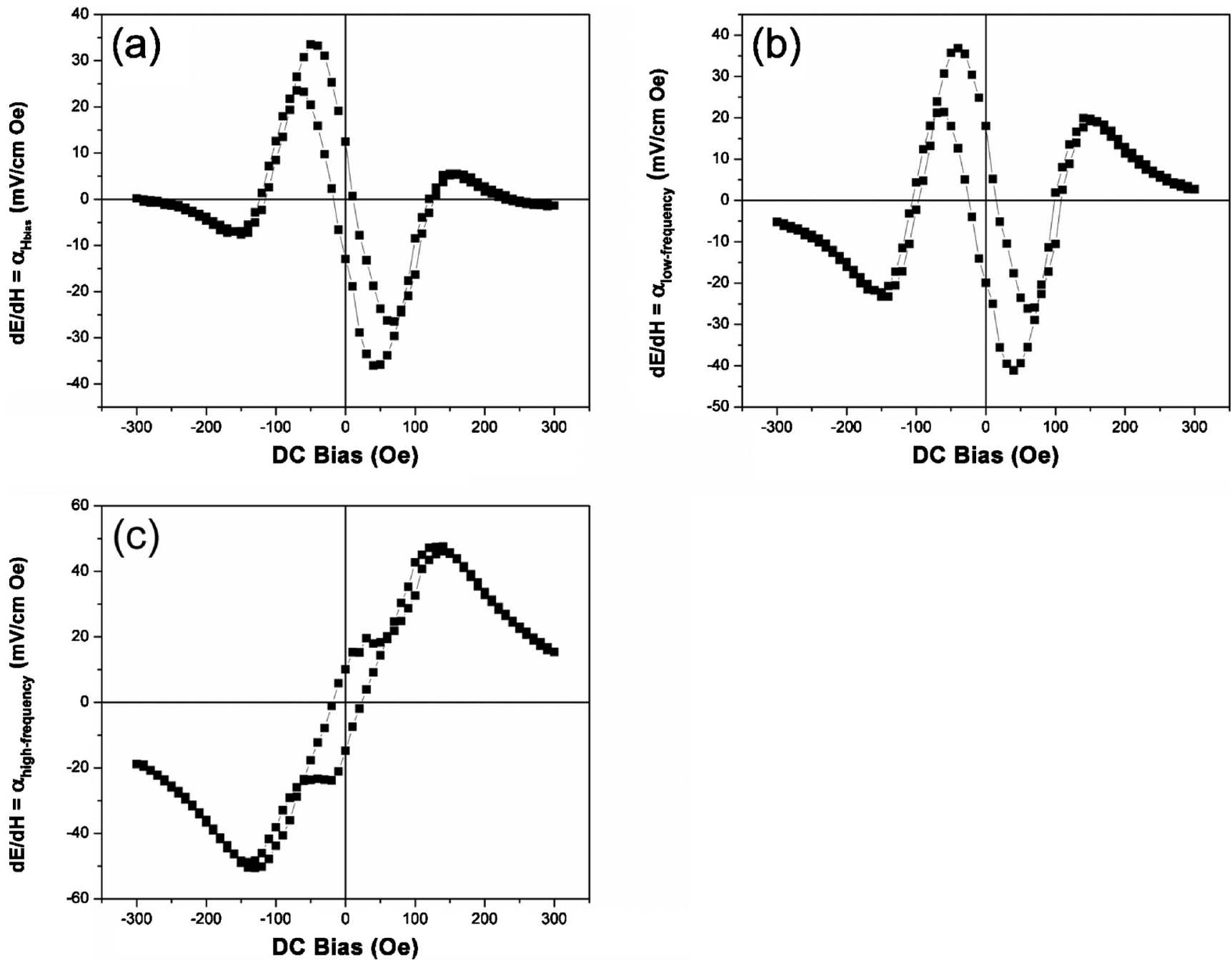

FIG. 5. ME voltage coefficients $\left(\alpha_{\mathrm{E}}\right)$ calculated by using measured values of NKNLS-NZF/Ni/NKNLS-NZF $\left(\alpha_{\text {bending strain }+\mathrm{H}_{\text {bias }}}\right)$ and NKNLS/Ni/NKNLS $\left(\alpha_{\text {bending strain }}\right)$ trilayer, (a) $\alpha\left(\mathrm{H}_{\text {bias }}\right)$, (b) $\alpha_{\text {low-frequency }}=\left(\alpha_{\text {bending strain }+\mathrm{H}_{\text {bias }}}+0.5 \alpha_{\mathrm{H}_{\text {bias }}}\right)$, and (c) $\alpha_{\text {high-frequency }}=\left(\alpha_{\text {bending strain }+\mathrm{H}_{\text {bias }}}+2.5 \alpha_{\text {bending strain }}\right)$.

shown in Fig. 4(d). It also illustrates that increased ME coefficient at magnetic dc bias of 169.3 Oe leads to only one zero-cross over point of $\alpha_{\mathrm{E}}$. This figure clearly shows the promise of this structure in providing the possible electrically tuned memory states, and leads us to the possibility of designing a magnetic field controlled memristor element.

The ME response of BMT as a function of frequency can be explained by using the measured values of $\alpha_{\mathrm{E}}$ for the NKNLS-NZF/Ni/NKNLS-NZF BMT and NKNLS/Ni/ NKNLS BMT laminates. Figures 5(a)-5(c) summarizes the role of magnetic interaction and bending strain on the hysteresis in ME voltage coefficients $\left(\alpha_{\mathrm{E}}\right)$. Figures 2(d) and 2(e) show the measured values of $\alpha_{\mathrm{E}}$ for NKNLS-NZF/Ni/ NKNLS-NZF BMT and NKNLS/Ni/NKNLS trilayer. Please note that the structure in Fig. 2(d) has NZF phase so there is magnetic interaction with $\mathrm{Ni}$, while that in Fig. 2(e) does not have any NZF phase. Besides this difference, the electrical connections and layer dimensions are exactly similar in both the cases. Comparing Figs. 2(d) and 2(e), one can immediately notice that $\mathrm{ME}$ hysteretic response requires the presence of magnetic interaction. Subtracting the magnitude of ME coefficient in Fig. 2(e) $\left(\alpha_{\text {bending strain }}\right)$ from that in Fig. 2(d) $\left(\alpha_{\text {bending strain }+\mathrm{H}_{\text {bias }}}\right)$, we can delineate the contribution arising from the magnetic interaction, or $\alpha\left(\mathrm{H}_{\text {bias }}\right)$ as shown in Fig. 5(a). Next, we try to understand the role of $\mathrm{H}_{\text {bias }}$ by increasing its contribution to the overall $\mathrm{ME}$ response. Figure 5 (b) plots the ME coefficient given as $\alpha_{\text {low-frequency }}$ $=\left(\alpha_{\text {bending strain }+\mathrm{H}_{\text {bias }}}+0.5 \alpha_{\mathrm{H}_{\text {bias }}}\right)$, which resembles the data shown in Figs. 4(a) and 4(b) at low frequencies. This indicates that by increasing the contribution related to magnetic interaction in proportion to that from bending strain, high remnant $\alpha_{\mathrm{ER}}$ and narrow hysteresis near zero dc bias can be induced. Figure 5(c) plots the ME coefficient given as $\alpha_{\text {high-frequency }}=\left(\alpha_{\text {bending strain }+\mathrm{H}_{\text {bias }}}+2.5 \alpha_{\text {bending strain }}\right)$, which resembles the behavior obtained at resonance frequency of $55.7 \mathrm{kHz}$ as shown in Fig. 4(d). These calculations show that a linear relation between the contributions from the magnetic interaction and bending strain leads to emergence of remanant $\alpha_{\mathrm{ER}}$ and zero-cross over point of $\alpha_{\mathrm{E}}$. The increase in contribution from the bending strain leads to increase in the magnitude of $\alpha_{\mathrm{E}}$ and suppresses the peaks at higher magnetic dc bias. As a result, the BMT's hysteresis and remnant $\alpha_{\mathrm{ER}}$ with first cross over point of $\alpha_{\mathrm{E}}$ dominantly depend on mag- 
netic interaction. On the other hand, bending strain is important for high magnitude of $\alpha_{\mathrm{E}}$ at higher dc bias with second cross over point.

\section{SUMMARY}

In summary, we report self-biased ME effects in leadfree three-phase laminates. The laminates consist of one piezoelectric phase and two ferromagnetic phases which couple with each other through differences in susceptibilities and coercive field. The self-biased ME response of NKNLSNZ/Ni/NKNLS-NZF trilayer laminates was shown to be combination of $\mathrm{ME}$ behavior of NKNLS-NZF/Ni/NKNLSNZF BMT and NKNLS/Ni/NKNLS trilayer composites.

\section{ACKNOWLEDGMENTS}

The authors gratefully acknowledge the financial support from National Science Foundation (Award No. 0757502) and Army Research Office (W911NF0910061).

${ }^{1}$ N. A. Hill, J. Phys. Chem. B 104, 6694 (2000).

${ }^{2}$ S. X. Dong, J. G. Bai, J. Y. Zhai, J. F. Li, G. Q. Lu, D. Viehland, S. J. Zhang, and T. R. Shrout, Appl. Phys. Lett. 86, 3 (2005).

${ }^{3}$ N. A. Spaldin and M. Fiebig, Science 309, 391 (2005).

${ }^{4}$ Y. H. Chu, L. W. Martin, M. B. Holcomb, M. Gajek, S. J. Han, Q. He, N.
Balke, C. H. Yang, D. Lee, W. Hu, Q. Zhan, P. L. Yang, A. FraileRodriguez, A. Scholl, S. X. Wang, and R. Ramesh, Nature Mater. 7, 478 (2008).

${ }^{5}$ C. W. Nan, M. I. Bichurin, S. X. Dong, D. Viehland, and G. Srinivasan, J. Appl. Phys. 103, 031101 (2008).

${ }^{6}$ J. Das, J. Gao, Z. Xing, J. F. Li, and D. Viehland, Appl. Phys. Lett. 95, 092501 (2009).

${ }^{7}$ J. Zhai, Z. Xing, S. Dong, J. Li, and D. Viehland, J. Am. Ceram. Soc. 91, 351 (2008).

${ }^{8}$ S. K. Mandal, G. Sreenivasulu, V. M. Petrov, and G. Srinivasan, Appl. Phys. Lett. 96, 192502 (2010)

${ }^{9}$ C. Sudakar, R. Naik, G. Lawes, J. V. Mantese, A. L. Micheli, G. Srinivasan, and S. P. Alpay, Appl. Phys. Lett. 90, 062502 (2007).

${ }^{10}$ J. V. Mantese, A. L. Micheli, N. W. Schubring, R. W. Hayes, G. Srinivasan, and S. P. Alpay, Appl. Phys. Lett. 87, 082503 (2005).

${ }^{11}$ S. N. Babu, K. Srinivas, and T. Bhimasankaram, J. Magn. Magn. Mater. 321, 3764 (2009).

${ }^{12}$ X. L. Zhong, M. Liao, J. B. Wang, S. H. Xie, and Y. C. Zhou, J. Cryst. Growth 310, 2995 (2008).

${ }^{13}$ S. J. Zhang, R. Xia, T. R. Shrout, G. Z. Zang, and J. F. Wang, J. Appl. Phys. 100, 6 (2006).

${ }^{14}$ R. A. Islam and S. Priya, Appl. Phys. Lett. 89, 152908 (2006).

${ }^{15}$ R. A. Islam and S. Priya, J. Mater. Sci. 43, 3560 (2008).

${ }^{16}$ C. S. Park, C. W. Ahn, S. C. Yang, and S. Priya, J. Appl. Phys. 106, 5 (2009).

${ }^{17}$ See supplementary material at http://dx.doi.org/10.1063/1.3493154 for impedance and phase spectra for bilayer and trilayer laminates under applied ac electric field, (a) NKNLS-NZF/Ni bilayer, (b) NKNLS/Ni bilayer, (c) NKNLS-NZF/Ni/NKNLS-NZF RMT, (d) NKNLS-NZF/Ni/NKNLS-NZF bending-mode laminate, and (e) NKNLS/Ni/NKNLS trilayer. 